\title{
Theoretical and Experimental Reflection Coefficients in Flexible Tubes as a Function of the Mach Number
}

\author{
Alessandro Giudici ${ }^{1}$, Wisam Hacham ${ }^{1,2}$, Ashraf W Khir ${ }^{1}$ \\ ${ }^{1}$ Brunel University London, Uxbridge, United Kingdom \\ ${ }^{2}$ University of Baghdad, Baghdad, Iraq
}

\begin{abstract}
The standard formulation of Wave Intensity Analysis (WIA) assumes that the flow velocity $(U)$ in the conduit is $<<$ the velocity of propagation of waves (c) in the system, and Mach number, $M=U / c$, is negligible. However, in the large conduit arteries, $U$ is relatively high due to ventricular contraction and $c$ is relatively low due to the large compliance; thus $M$ is $>0$, and may not be ignored. Therefore, the aim of this study is to identify experimentally the relationship between $M$ and the reflection coefficient in vitro.

Combinations of flexible tubes, of $2 \mathrm{~m}$ in length with isotropic and uniform circular cross sectional area along their longitudinal axes, were used to present mother and daughter tubes to produce a range of reflection coefficients. An approximately semi-sinusoidal pulse was generated at the inlet of the mother tube using a syringe pump, first in the condition of initial velocity, $U_{0}=0$, and when $U_{0}>0$ with steady flow to superimpose the pulse.

Pressure $(P)$ and Velocity $(U)$ were measured in the mother tube, wave speed was determined using the foot to foot and PU-loops methods. The theoretical reflection coefficient, $R_{t}$ at $M=0$, has been compared to the experimental reflection coefficient, $R$ at $M>0$, which was determined as $d P-/ d P+$ as calculated using WIA.

The function $R(M)$ changes significantly with the geometrical and mechanical features of the connected tubes. In our experiments, $R$ increased significantly with small values of $M$. In the range of $M=0-0.02, R$ increased by $4-36 \%$. Therefore, we conclude that $M$ significantly affects the magnitude of reflections.
\end{abstract}

\section{Introduction}

Arterial reflected waves play an important role in determining the features of the arterial pressure waveforms, including systolic pressure $(1,2)$. Also, arterial aging and pathologies (e.g. hypertension) affect both the magnitude and timing of arterial reflected waves and, therefore, the features of the pressure waveform $(3,4)$.
The magnitude of reflected waves can be quantified by means of the reflection coefficient; i.e. the ratio between the backward to forward components of the pressure waveform or of the wave intensity. This parameter is normally assumed to be independent of the mean value of blood velocity in the artery. In fact, to derive the standard WIA formulation the blood velocity is assumed to be $<<$ wave speed in arteries $(\mathrm{M} \approx 0)$, and, therefore, the convective component is neglected (5). While this assumption maybe reasonable in a considerable portion of the arterial tree, large conduit arteries, such as the aorta and pulmonary artery, present relatively low wall stiffness and relatively high blood velocity, neglecting the convective term may induce errors in the estimation of wave reflections. Therefore, the aim of the present work is to determine the effect of $\mathrm{M}$ on the reflection coefficient in flexible tubes in vitro.

\section{Methods}

\subsection{Experimental setup}

The experimental setup is shown in Fig. 1.

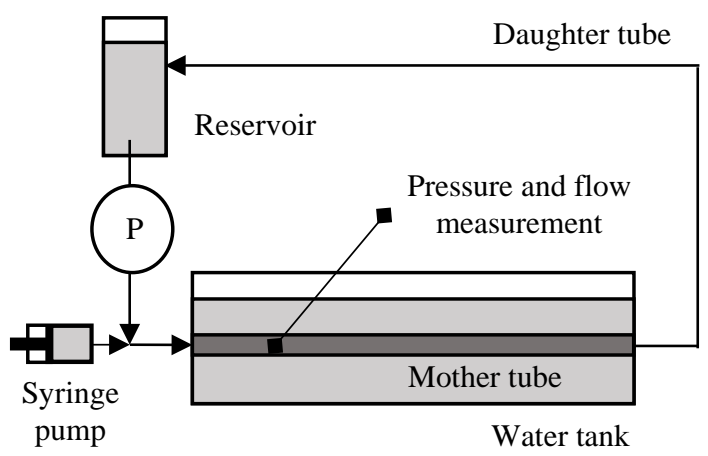

Figure 1 - Schematic representation of the experimental setup. Pressure was recorded using a transducer tipped catheter, and flow using an ultrasound probe. All the components were located on a horizontal plane. 


\subsubsection{Tubes}

We used five 2 meters long tubes as mother tubes and four $>9$ meters long tubes of different size and material as daughter tubes. The length of the daughter tube was necessary to isolate the reflection generated at the connection between mother and daughter tubes from that generated at the connection between daughter tube and reservoir. Mechanical properties of the tubes are included in Table 1, and the geometrical and mechanical properties of each tube were uniform along its longitudinal axis. $c$ has been determined by averaging the results obtained with the foot-to-foot and PU-loop techniques.

The connection between mother and daughter tubes was realized overlapping them of approx. $1-2 \mathrm{~cm}$. If the difference in diameter was too high, a third tube was inserted between mother and daughter to allow the connection.

Table 1 - Properties of the tubes used in the experiment, Din: internal diameter, h: wall thickness, c: waves speed.

\begin{tabular}{cccc}
\hline $\mathbf{D}_{\text {in }}[\mathrm{mm}]$ & $\mathbf{h}[\mathrm{mm}]$ & Material & $\mathbf{c}[\mathrm{m} / \mathrm{s}]$ \\
\hline $\mathbf{8}$ & 2 & Silicone & 25.02 \\
\hline $\mathbf{9 . 5 2 5}$ & 0.8 & Latex & 12.70 \\
\hline $\mathbf{1 0}$ & 1 & Silicone & 21.52 \\
\hline $\mathbf{1 7}$ & 1.5 & Rubber & 20.76 \\
\hline $\mathbf{1 9 . 0 5}$ & 2.4 & Latex & 14.17 \\
\hline $\mathbf{2 0 . 6}$ & 1.7 & Rubber & 20.38 \\
\hline
\end{tabular}

\subsubsection{Pumps}

Two pumps were used for this experiment: a piston pump, producing an approximately single semi-sinusoidal pulse, and a centrifugal pump providing a continuous flow. The speed of the centrifugal pump was adjusted to vary the flow; needed to provide a variation of $\mathrm{M}$.

\subsubsection{Measurements}

Simultaneous pressure and flow were measured at the same axial location (52 $\mathrm{cm}$ from the inlet of the mother tube). Pressure was measured using a $6 \mathrm{~F}$ pressure transducer tipped catheter (Millar, Inc, Huston, Texas, USA), and flow was measured using ultrasound flow probes (Transonic System, NY, USA). Data were sampled at $500 \mathrm{~Hz}$.

\subsection{Theoretical reflection coefficient}

When $M \approx 0$, the theoretical reflection coefficient, $R_{t}$, characterizing the connection between the mother and daughter tubes is calculated as follows:

$$
R_{t}=\frac{\frac{A_{1}}{c_{1}}-\frac{A_{2}}{c_{2}}}{\frac{A_{1}}{c_{1}}+\frac{A_{2}}{c_{2}}}
$$

where $\mathrm{A}$ and $\mathrm{c}$ are the internal sectional areas and wave speeds of the tubes, respectively, and the subscripts $(1,2)$ indicate the mother and daughter tubes, respectively.

\subsection{Experimental reflection coefficient}

The standard formulation of WIA was used to separate the pressure and velocity waveforms in their forward and backward components (6). The experimental reflection coefficient (R) was calculated as the ratio of the peak of the backward to forward component of the separated pressure waveform:

$$
R=\frac{d P_{-}}{d P_{+}}
$$

\subsection{Data analysis}

For each connection configuration, pressure and velocity waveforms were sampled and the data used in the calculations are the average of 7 recordings. The rotational speed of the centrifugal pump was varied between 0 and the maximum flow (which depended on the resistance provided by the mother and daughter tubes connected downstream). The whole range of flows was divided into 6-8 steps and $\mathrm{P}$ and $\mathrm{U}$ recordings were performed for each step.

Collected data were analyzed with a Matlab code (The Mathworks, Natick, MA, USA). The value of the reflection coefficient for each $\mathrm{M}$ was calculated averaging the results obtained in the 7 repeated measurements.

\section{Results}

\subsection{Reflection coefficient at $M=0$}

Table 2 shows a comparison between $\mathrm{R}_{\mathrm{t}}$ calculated with (1) and $R_{0}(R$ when $M=0)$ determined experimentally for each connection configuration used in the present work.

\section{2. $\quad \mathrm{R}$ as a function of $\mathbf{M}$}

With all the connection configurations, an increase of $\mathrm{M}$ resulted in an increase of $\mathrm{R}$ (Figure 2). This was found for both negative and positive $R_{t}$, with a strong correlation between $\mathrm{R}$ and $\mathrm{M}$. A fitting of $\mathrm{R}(\mathrm{M})$ provided an $\mathrm{R}^{2}>0.92$ (linear fitting). Interestingly, the percentage of increase of $\mathrm{R}$ at a given $\mathrm{M}$ was different in different connection configurations as indicated by the slope (Table 2). For example, at $\mathrm{M} \approx 0.02$, $\mathrm{R}$ increased by $50 \%$ in connection $\mathrm{D}$, by $30 \%$ in $\mathrm{H}$ and by $10 \%$ in connection $\mathrm{F}$.

\section{Discussion}

The results showed a good agreement between the theoretical and the experimental reflection coefficients at 


\begin{tabular}{cccccc}
\hline Connection & Mother $\boldsymbol{\varnothing}[\mathrm{mm}]$ & Daughter $\boldsymbol{\varnothing}[\mathrm{mm}]$ & $\mathbf{R}_{\mathbf{t}}$ & $\mathbf{R}_{\mathbf{0}}$ & \multicolumn{2}{c}{ Slope (95\% C.I.) } \\
\hline $\mathbf{A}$ & 20.6 & 8 & 0.78 & $0.696 \pm 0.013$ & $8.7-25.9$ \\
\hline $\mathbf{B}$ & 17 & 8 & 0.697 & $0.694 \pm 0.010$ & $12.3-14.0$ \\
\hline $\mathbf{C}$ & 19.05 & 8 & 0.817 & $0.761 \pm 0.011$ & $10.9-16.3$ \\
\hline $\mathbf{D}$ & 10 & 8 & 0.290 & $0.285 \pm 0.005$ & $5.6-8.8$ \\
\hline $\mathbf{E}$ & 17 & 10 & 0.499 & $0.505 \pm 0.004$ & $6.3-8.2$ \\
\hline $\mathbf{F}$ & 19.05 & 19.6 & 0.105 & $0.076 \pm 0.011$ & $2.8-4.5$ \\
\hline $\mathbf{G}$ & 9.525 & 10 & 0.212 & $0.206 \pm 0.006$ & $2.1-2.7$ \\
\hline $\mathbf{H}$ & 9.525 & 8 & 0.473 & $0.531 \pm 0.008$ & $2.2-3.4$ \\
\hline $\mathbf{I}$ & 10 & 19.6 & -0.635 & $-0.605 \pm 0.009$ & $2.1-3.4$ \\
\hline $\mathbf{J}$ & 10 & 17 & -0.499 & $-0.465 \pm 0.008$ & $2.1-3.7$ \\
\hline
\end{tabular}

Table 2 - Comparison between the theoretical reflection coefficient $R_{t}$ and the experimentally determined $R_{0}$ at $M_{=0}$. $R_{0}$ is expressed as mean $\pm \mathrm{SD}$.
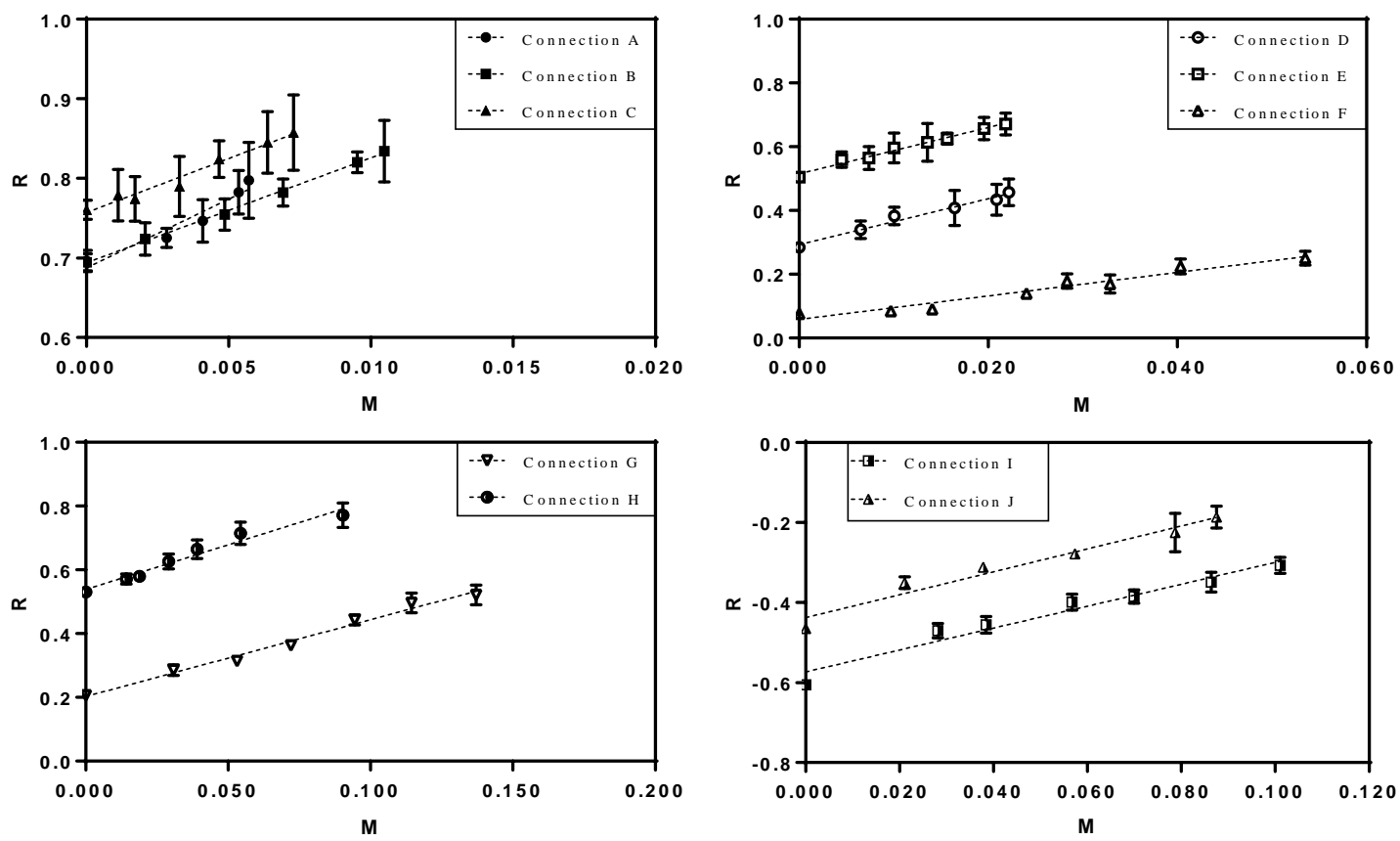

Figure 2 - $\mathrm{R}$ as a function of $\mathrm{M}$ for all the connection described in table 2. Data are presented as mean and error bars indicate SD.

initial $\mathrm{U}=0$. The largest error was found for connection $\mathrm{A}$ $\left(\mathrm{R}_{\mathrm{t}}=0.78\right.$ and $\left.\mathrm{R}_{0}=0.70\right)$. The theoretical reflection coefficient assumes an ideal connection between tubes with no overlapping. On the other hand, the real connection between tubes may have introduced a deviation of $\mathrm{R}_{0}$ from $\mathrm{R}_{\mathrm{t}}$.

All the connections used in this study showed an increase in $\mathrm{R}$ with increasing $\mathrm{M}$. However, this finding has different implications for positive and negative reflections. In the case of positive reflections, the superimposed steady state flow enhances the discontinuity given by the connection between mother and daughter tubes. On the other hand, the amplitude of negative reflections is decreased by increasing $\mathrm{M}$, with $\mathrm{R}$ approaching 0 . Therefore, positive and negative reflections present an opposite behavior with increasing $\mathrm{M}$.

Interestingly, the slope of the linear fitting was not equal for all the connections. It seems the slopes depends on the relative geometrical and mechanical properties of the connected tubes. These results indicate that higher values $\mathrm{R}_{0}$ produce higher slopes of $\mathrm{R}$ as a function of $\mathrm{M}$. However, connection $\mathrm{H}$ shows a significantly lower slope with respect to $\mathrm{D}$ and $\mathrm{E}$ but has similar $\mathrm{R}_{0}$, and comparable to connection $\mathrm{F}$ and $\mathrm{G}$ ( $\mathrm{R} \leq 0.2$ for $\mathrm{M}=0$ ). Therefore, other elements seem to play a role in determining the trend of $\mathrm{R}$ as a function of $\mathrm{M}$. For example, connection A, B, C, D, and $\mathrm{E}$ were made between silicone or rubber tubes having similar values of wall stiffness ( $c=20-25 \mathrm{~m} / \mathrm{s}$ ), while connection $\mathrm{F}, \mathrm{G}$, and $\mathrm{H}$ were between latex and silicone/rubber, with latex being significantly more compliant ( $c=12-15 \mathrm{~m} / \mathrm{s}$ ). This could possibly explain the difference between $\mathrm{H}$ and $\mathrm{D}-\mathrm{E}$. The slopes of the normalized reflection coefficient $\left(R / R_{0}\right)$ have also been compared. A significant difference was found between all the connections. Figure 3 (top), a different slope can be identified between connection A and $\mathrm{E}$ even for very low values of $\mathrm{M}\left(\approx 10^{-2}\right)$. 

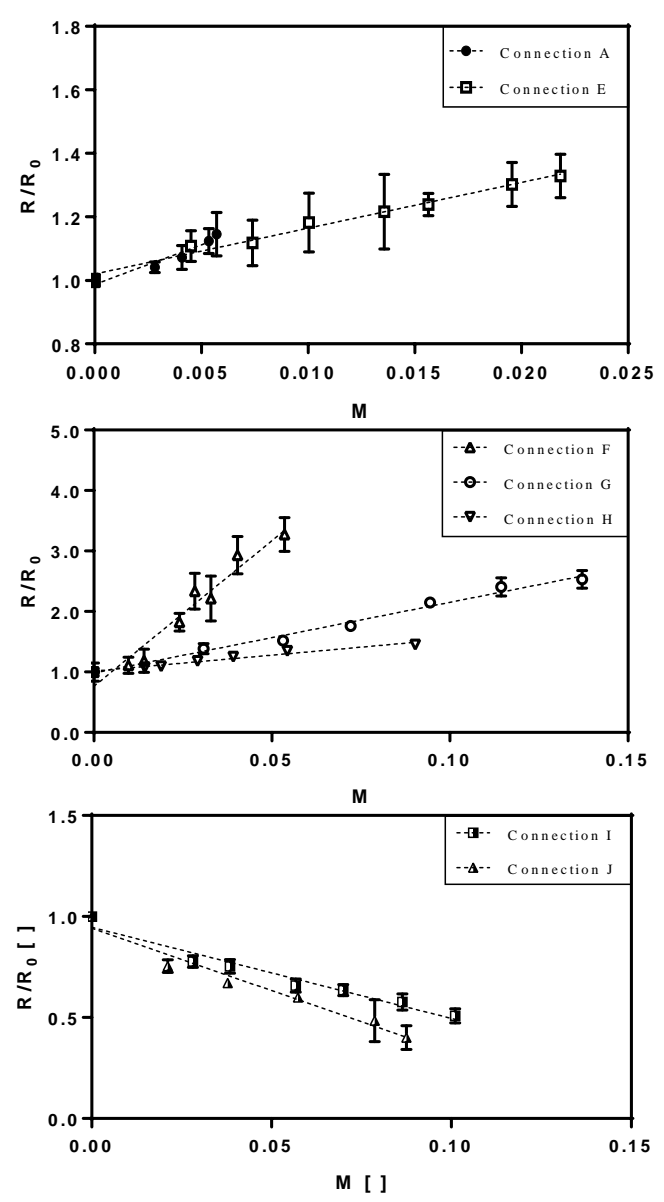

Figure 3 - Normalized reflection coefficient $\left(\mathrm{R} / \mathrm{R}_{0}\right)$ as a function of M. Data are presented as mean (SD).

It is worth considering that $\mathrm{R}$ is bounded between -1 and 1 by definition, since $P_{i}=P_{t}-P_{r}$, where $P_{i}$ is the incident pressure wave, $\mathrm{P}_{\mathrm{t}}$ is the transmitted pressure wave and $\mathrm{P}_{\mathrm{r}}$ is the reflected pressure wave. Therefore $R(M)$ is expected to reach a plateau at high values of $M$. However, this trend was not shown in the range of $M$ used in our results, except for negative reflections. When fitting the results with $2^{\text {nd }}$ order polynomial functions, the sign of the quadratic term was negative only in 4 of the connection configurations with positive $\mathrm{R}$.

The dependence of $\mathrm{R}$ on $\mathrm{M}$ becomes relevant when studying wave propagation in large conduit arteries. In the main pulmonary artery, $\mathrm{M}$ is significantly higher in healthy subjects than in hypertensive $(0.071 \pm 0.045$ and $0.023 \pm$ 0.019 , respectively) due to the pathological arterial stiffening (using data collected in (7)). Therefore, when comparing the two groups, the experimental $\mathrm{R}$ may not completely describe the remodelling process caused by hypertension and the effect of $\mathrm{M}$ has also to be taken into account.

One of the limitations of this work is the lack of high values of $\mathrm{M}$. This might have been due to the needed lengthy daughter tube, which introduced a high hydraulic resistance, limiting the flow rate produced by the continuous flow pump.

\section{Conclusion}

In our experiments, $\mathrm{R}$ is dependent on $\mathrm{M}$, and a significant alteration of $\mathrm{R}$ can be obtained for $\mathrm{M}<0.05$. Moreover, the function R(M) seems to be dependent on the relative geometrical and mechanical properties of the connected mother and daughter tubes. This could be relevant when considering waves reflections in large conduit arteries and the alteration of $\mathrm{R}$ with arterial pathologies.

More work is needed to define a mathematical formulation to describe the dependency of $\mathrm{R}$ from $\mathrm{M}$ and to explain the different behavior of different types of connections.

\section{References}

[1] Westerhof N, Sipkema P, van den Bos, G C, Elzinga G. Forward and backward waves in the arterial system. Cardiovasc Res. 1972;6(6):648-56.

[2] Khir AW, Parker KH. Wave intensity in the ascending aorta: effects of arterial occlusion. Journal of Biomechanics. 2005;38(4):647-55.

[3] Borlotti A, Khir AW, Rietzschel ER, De Buyzere ML, Vermeersch S, Segers P. Noninvasive determination of local pulse wave velocity and wave intensity: changes with age and gender in the carotid and femoral arteries of healthy human. Journal of Applied Physiology. 2012 Sep;113(5):727-35.

[4] Su J, Manisty C, Simonsen U, Howard LS, Parker KH, Hughes AD. Pulmonary artery wave propagation and reservoir function in conscious man: impact of pulmonary vascular disease, respiration and dynamic stress tests. The Journal of Physiology. 2017 Oct 15;595(20):6463-76.

[5] Parker K. An introduction to wave intensity analysis. Med Biol Eng Comput. 2009 Feb;47(2):175-88.

[6] Parker KH, Jones CJH. Forward and backward running waves in the arteries: Analysis using the method of characteristics. J Biomech Eng. 1990;112(3):322-6.

[7] Su j, Manisty C, Parker KH, Simonsen U, Nielsen-Kudsk JE, Mellemkjaer S, Connolly S, Lim PB, Whinnett ZI, Malik IS, Watson G, Davies JE, Gibbs S, Hughes AD, Howard L. Wave intensity analysis provides novel insights into pulmonary arterial hypertension and chronic thromboembolic pulmonary hypertension. J Am Heart Assoc. 2017 Sep;6(11).

Address for correspondence.

Ashraf W. Khir.

Brunel University London, Kingston Lane, Uxbridge, United Kingdom, UB8 3PH.

Ashraf.Khir@brunel.ac.uk 